\title{
Genetic Polymorphisms in NAT1, NAT2, GSTM1, GSTP1 and GSTT1 and Susceptibility to Colorectal Cancer among Filipinos
}

Eva Maria C. Cutiongco-de la Paz, ${ }^{1,2}$ Corazon A. Ngelangel, ${ }^{3}$ Virgilio P. Bañez, ${ }^{4}$ Francisco T. Roxas, ${ }^{5}$ Catherine Lynn T. Silao, ${ }^{1,19}$ Jose B. Nevado Jr., ${ }^{1}$ Alberto B. Roxas, ${ }^{5}$ Oliver G. Florendo, ${ }^{4}$ Ma. Cecilia M. Sison, ${ }^{4}$

Orlino Bisquera, Jr., ${ }^{5}$ Luminardo M. Ramos, ${ }^{5}$ Elizabeth A. Nuqui, ${ }^{6}$ Arnold Joseph M. Fernandez, ${ }^{6}$

Maria Constancia O. Carrillo, ${ }^{7}$ Beatriz J. Tiangco, ${ }^{1,8}$ Aileen D. Wang, ${ }^{9}$ Rosalyn H. Sebastian, ${ }^{9}$ Richmond B. Ceniza, ${ }^{9}$

Leander Linus Philip P. Simpao, ${ }^{9}$ Lakan U. Beratio, ${ }^{9}$ Eleanor A. Dominguez, ${ }^{9}$ Albert B. Albay Jr., ${ }^{9}$

Alfredo Y. Pontejos Jr., ${ }^{10}$ Nathaniel W. Yang, ${ }^{10}$ Arsenio A. Cabungcal, ${ }^{10}$ Rey A. Desales, ${ }^{11}$ Nelia S. Tan-Liu,,${ }^{11}$ Sullian S. Naval, ${ }^{11}$ Roberto M. Montevirgen, ${ }^{11}$ Catalina de Siena E. Gonda-Dimayacyac, ${ }^{11}$ Pedrito Y. Tagayuna, ${ }^{11}$

John A. Coloma, ${ }^{12}$ Gil M. Vicente, ${ }^{12}$ Higinio T. Mappala, ${ }^{12}$ Alex C. Tapia, ${ }^{12}$ Emmanuel F. Montana Jr., ${ }^{12}$ Jonathan M. Asprer, ${ }^{12}$ Reynaldo O. Joson, ${ }^{13}$ Sergio P. Paguio, ${ }^{13}$ Tristan T. Chipongian, ${ }^{14}$ Joselito F. David, ${ }^{14}$

Florentino C. Doble, ${ }^{14}$ Maria Noemi G. Pato, ${ }^{14}$ Benito B. Bionat Jr., ${ }^{15}$ Hans Francis D. Ferraris, ${ }^{15}$

Adonis A. Guancia, ${ }^{15}$ Eriberto R. Layda, ${ }^{15}$ Andrew D. Dimacali, ${ }^{16}$ Conrado C. Cajucom, ${ }^{16}$ Richard C. Tia, ${ }^{16}$ Mark U. Javelosa, ${ }^{3}$ Regie Lyn P. Santos-Cortez, ${ }^{17,18}$ Frances Maureen C. Rocamora, ${ }^{1}$ Roemel Jeusep Bueno ${ }^{1}$ and Carmencita D. Padilla ${ }^{1,2,19}$

\author{
${ }^{1}$ Institute of Human Genetics, National Institutes of Health, University of the Philippines Manila \\ ${ }^{2}$ Philippine Genome Center, University of the Philippines System \\ ${ }^{3}$ Institute of Clinical Epidemiology, National Institutes of Health, University of the Philippines Manila \\ ${ }^{4}$ Section of Gastroenterology, Department of Medicine, College of Medicine and Philippine General Hospital, University of the Philippines Manila \\ ${ }^{5}$ Department of Surgery, College of Medicine and Philippine General Hospital, University of the Philippines Manila \\ ${ }^{6}$ Department of Pathology, College of Medicine, University of the Philippines Manila \\ ${ }^{7}$ College of Arts and Sciences, University of the Philippines Manila \\ ${ }^{8}$ National Kidney and Transplant Institute \\ ${ }^{9}$ Section of Pulmonary Medicine, Department of Medicine, College of Medicine and Philippine General Hospital, University of the Philippines Manila \\ ${ }^{10}$ Department of Otorhinolaryngology, College of Medicine and Philippine General Hospital, University of the Philippines Manila \\ ${ }^{11}$ Lung Center of the Philippines \\ ${ }^{12}$ Jose R. Reyes Memorial Medical Center \\ ${ }^{13}$ Ospital ng Maynila \\ ${ }^{14}$ East Avenue Medical Center \\ ${ }^{15}$ Corazon Locsin Montelibano Memorial Regional Hospital \\ ${ }^{16}$ Medical Center Manila \\ ${ }_{17}$ University of Colorado School of Medicine, Colorado, USA \\ ${ }^{18}$ Philippine National Ear Institute, National Institutes of Health, University of the Philippines Manila \\ ${ }^{19}$ Department of Pediatrics, College of Medicine and Philippine General Hospital, University of the Philippines Manila
}

\begin{abstract}
Objectives. Polymorphisms in metabolic genes which alter rates of bioactivation and detoxification have been shown to modulate susceptibility to colorectal cancer. This study sought to evaluate the colorectal cancer risk from environmental factors and to do polymorphism studies on genes that code for Phase I and II xenobiotic metabolic enzymes among Filipino colorectal cancer patients and matched controls.
\end{abstract}

Corresponding authors: Eva Maria Cutiongco-de la Paz, MD and Carmencita D. Padilla, MD, MAHPS

Institute of Human Genetics

National Institutes of Health

University of the Philippines Manila

623 Pedro Gil St., Ermita, Manila 1000 Philippines

Telephone: +6325264266

Email: eccutiongcodelapaz@up.edu.ph
Methods. A total of 224 colorectal cancer cases and 276 controls from the Filipino population were genotyped for selected polymorphisms in GSTM1, GSTP1, GSTT1, NAT1 and NAT2. Medical and diet histories, occupational exposure and demographic data were also collected for all subject participants.

Results. Univariate logistic regression of non-genetic factors identified exposure to UV (sunlight) (OR 1.99, 95\% Cl: 1.16-3.39) and wood dust (OR 2.66, 95\% Cl: 1.21-5.83) and moldy food exposure (OR 1.61, 95\% Cl:1.11-2.35) as risk factors; while the NAT2*6B allele (recessive model OR 1.51, 95\% Cl :1.06-2.16; dominant model OR 1.87, 95\% Cl: 1.05-3.33) and homozygous genotype (OR 2.19, 95\% Cl: 1.19-4.03) were found to be significant among the genetic factors. After multivariate logistic regression of both environmental and genetic factors, only UV radiation exposure (OR 2.08, 95\% Cl: 1.21-3.58) and wood dust exposure (OR 2.08, 95\% Cl: 0.95-5.30) remained to be 
significantly associated with increasing colorectal cancer risk in the study population.

Conclusion. This study demonstrated that UV sunlight and wood dust exposure play a greater role in influencing colorectal cancer susceptibility than genotype status from genetic polymorphisms of the GST and the NAT' genes.

Key Words: colorectal cancer, genetic polymorphisms, xenobiotic metabolizing enzymes, Filipino, susceptibility

\section{Introduction}

Colorectal cancer is one of the leading cancers of the world. Based on incidence, it ranks fourth in men and third in women, with over one million new cases worldwide. ${ }^{1}$ The majority $(>90 \%)$ of cancers of the colon and rectum are adenocarcinomas. ${ }^{2}$ In the Philippines, the Philippine Society of Gastroenterology (PSG) stated that colorectal cancer is the number one cancer in the country as of March 2017. The data of PSG showed that among Filipinos, there are about 3000 new cases of colorectal annually and $67 \%$ of the reported cases die. ${ }^{3}$

The development of colon cancer can usually occur in one of three patterns: hereditary, familial and sporadicmajority of colorectal cancer cases (70-75\%), however, are sporadic. ${ }^{1}$ Disease etiology has been linked to both genetic and non-genetic factors. Factors pertaining to diet (intake of red/processed meats) and lifestyle (alcohol intake, obesity and physical inactivity) have been shown in several studies to be associated with risk for this disease. ${ }^{4-9}$

Population-based studies on different polymorphic genes have also shown that genetic variations influence susceptibility to certain types of cancer. Genes of interest include those that code for phase I and phase II xenobiotic metabolizing enzymes such as $\mathrm{N}$-acetyltransferases (NATs) and Glutathione-S-Transferases (GSTs). NATs and GSTs are responsible for metabolizing diet- and environmentallyderived colorectal carcinogens such as heterocyclic and aromatic amines, and polyaromatic hydrocarbons. $6,7,10$ Heritable variations in such genes occurring as genetic polymorphisms can result in functional and allelic variants that lead to different rates of metabolic activation or detoxification of particular carcinogens thereby resulting in varied cancer susceptibility among individuals. ${ }^{9,10}$

In humans, N-acetyltransferases (NATs) can be divided into two functional classes: NAT1 and NAT2. NAT1 and NAT2 perform N-acetylation, O-acetylation, and $\mathrm{N}$, Oacetyltransfer which activate or deactivate aromatic and heterocyclic amines. ${ }^{6,7}$ NAT1 is ubiquitous and found in almost all tissues while NAT2 is primarily present in the liver. ${ }^{11}$ Polymorphic variants of NAT1 and NAT2 give different rates of acetylation; slow acetylators (g.1095C $>$ A for NAT1; g.341T>C, g.481C $>\mathrm{T}$, g.803A $>\mathrm{G}$, g.590G $>\mathrm{A}$, and g.857G $>$ A for NAT2) and fast acetylators (g.1088T $>$ A and g.1095C>A, and 9del1065-1090 for NAT1). ${ }^{11,12}$ The rapid acetylator phenotype has been associated with increased susceptibility to colorectal cancer. ${ }^{4,7,9}$ Similarly, significant associations have also been found between NAT1 and NAT2 rapid acetylator genotypes and colorectal cancer risk in previous population-based studies. ${ }^{13-15}$ Both of these genes were also found to be downregulated in colorectal cancer patients. The low expression of the genes initiates and aids in the development of colorectal cancer. ${ }^{16}$

Another gene superfamily investigated in the study includes the Glutathione-S-transferase genes (GSTM1, GSTP1, GSTT1), which code for both cytosolic and membrane-bound proteins that are primarily involved in cellular detoxification by neutralizing the electrophilic sites of endogenous and exogenous compounds through conjugation with glutathione. This mechanism prevents the attack of potential reactive electrophiles on macromolecules. ${ }^{17,18}$ For the GSTT1 and GSTM1 genes, a polymorphic gene deletion which results in a lack of conjugation activity has been hypothesized to influence cancer initiation as a result of an impaired ability to detoxify electrophilic carcinogens. An increase in colorectal cancer risk has been reported previously among individuals having a homozygous deletion of GSTM1 and GSTT1. ${ }^{19-25}$ For GSTP1, the c.313A $>\mathrm{G}$ polymorphism has also been noted to be a significant modifier for colorectal cancer susceptibility. ${ }^{26}$ Previous studies have demonstrated that this particular polymorphism - which causes an Ile105Val substitution in the encoded product - exhibit altered catalytic activity and structural stability which affect over-all metabolic detoxification, contributing to individual cancer susceptibility. ${ }^{27,28}$

This study focused on genetic polymorphisms of the GST and the NAT genes as possible modifiers of colorectal cancer risk in the Filipino population. More specifically, this study sought to accomplish the following: a) to evaluate the colorectal cancer risk from environmental factors in addition to past medical, family, reproductive, and socio-demographic history; b) to do polymorphism studies on Phase I and II metabolic enzymes - GSTP1, GSTT1, GSTM1, NAT1 and NAT2 in colorectal cancer patients and their controls; and c) to evaluate the colorectal cancer risk from specific polymorphisms, comparing cancer cases and their controls.

\section{Methods}

\section{Subject Population}

This case-control study is comprised of 224 confirmed colorectal cancer cases, and 276 healthy controls. The patients were recruited from five tertiary hospitals (Philippine General Hospital, Ospital ng Maynila Medical Center, East Avenue Medical Center, Jose Reyes Memorial Medical Center and Medical Center Manila. The inclusion criteria were the presence of histopathologically confirmed colorectal cancer (any age, any stage, any Eastern 
Cooperative Oncology Group) and no history of chemotherapy or radiotherapy prior to his/her enrolment to the study. The inclusion criterion for the controls was the absence of any prior history of cancer. The controls (age-5 years-interval-matched; clinically free of cancer; sexmatched) were randomly selected from the same hospitals as the cases during the same time period. Classification was verified by obtaining the histopathology result of the case patient's tissue and blood was taken for genetic analysis. Ethical committee approval was obtained prior to study initiation and informed consents from the subject participants were acquired.

\section{Data Collection}

All subject participants were interviewed by trained health workers using a questionnaire and standardized interview and measurement techniques. Information including age, gender, occupation, tobacco and betel nut chewing habits, family history of colorectal cancer, oral contraceptive use, diet (consumption of alcohol, canned meat, fish sauce (patis), shrimp paste (bagoong), vegetables, scalding hot food, preserved foods, smoked foods, salted foods), and occupational exposure (exposure to moldy food, pesticides, vinyl chloride, benzene, UV sunlight, coal carbonization, and wood dust). The questionnaire and the interview technique were pre-tested among a group of Filipino patients and were modified accordingly. All data collected from the subject participants were treated as confidential.

\section{DNA Isolation}

Genomic DNA was extracted from whole blood samples of enrolled patients using the QIAamp® Blood Midi Kit Spin Protocol (Qiagen GmbH, Hilden, Germany). The DNA extracts were subsequently stored at $-20^{\circ} \mathrm{C}$.

\section{Genotyping}

Polymorphisms for GSTM1, GSTT1, GSTP1, NAT1 and NAT2 genes were detected using polymerase chain reaction (PCR) and PCR-restriction fragment length polymorphism (RFLP). Genotyping methods by PCR-RFLP were verified by direct sequencing.

GSTM1 and GSTT1. The presence of at least one allele of GSTM1 and GSTT1 was determined using standard PCR protocol. $^{28,29}$ As an internal control, exon 7 of the CYP1A1 gene was co-amplified for each reaction using previously published primers. ${ }^{29}$

GSTP1. The c.313A $>\mathrm{G}$ polymorphism in exon 5 was ascertained using PCR-RFLP with Alw26I. ${ }^{30}$

NAT1. Detection of NAT1 alleles was performed using PCR-RFLP with MboII for $N A T 1^{*} 4$, and $N A T 1^{*} 11$. Allele- specific PCR was performed using NAT1*3-specific and $N A T 1^{*} 10$-specific primers to distinguish between the two alleles. ${ }^{31}$ As an internal control for allele-specific PCR, $\beta$ globin was co-amplified using previously published primers.

NAT2. The PCR-RFLP strategy by Hubbard et al. was used to genotype NAT2.32

\section{Statistical Analysis}

For the environmental variables, statistical analysis was done using Stata Program version 9.0, while SPSS 14.0 software was used for statistical analysis of genetic variables. Age-and sex-matched pairs were analyzed including multiple controls per case within an age-group. Comparison of characteristics between groups was carried out through $\chi^{2}$ tests for genetic variables, McNemar's test for environmental variables, and independent t-tests for continuous (environmental) variables. Univariate conditional logistic regression analyses for environmental and genetic variables were initially carried out separately using simple conditional logistic regression to assess the significance of each independent variable or risk factor for cancer by cancer site. All environmental factors that were significant at 0.2 level in the univariate analyses are identified as the initial set of candidate predictors for colorectal cancer. They are then included in the multivariate conditional logistic regression model to generate adjusted ORs and corresponding p-values. All environmental factors that were significant in the multivariate analysis were included in multivariate logistic regression with statistically significant genetic variables (with ORs that are significant at $\alpha=0.05$ ) to test for geneenvironment interactions.

For diallelic genes GSTP1, CYP1A1 and CYP2E1 genotypes were tested for Hardy-Weinberg Equilibrium (HWE). Only four of 26 known NAT1 alleles and six of 62 known NAT2 alleles were tested, thus genotype frequencies for both genes are expected to deviate from HWE.

\section{Results}

A total of 500 patients with 224 cases and 276 controls were available for analysis (Table 1). Among the cases, there were 131 males and 93 females. The control population had 131 males and 145 females. Median age range for the whole group was 50-54 years old; 50-54 for the controls and 55-59 for the cases. Univariate logistic regression analyses of nongenetic factors identified exposure to moldy food $(\geq 1 /$ month) (OR 1.61; 95\% CI 1.11-2.35), UV sunlight exposure ( $\geq 7 \mathrm{x} /$ month) (OR 1.99; 95\% CI 1.16-3.39), and wood dust occupational exposure (OR 2.66; 95\% CI 1.21-5.83) as significant risk factors (Table 2). These three variables remained significant environmental risk factors after multivariate analysis (Table 3). Among the genetic factors, 
Table 1. Age and sex distribution of colorectal cancer cases and controls

\begin{tabular}{cccccc}
\hline \multirow{2}{*}{ Age } & \multicolumn{2}{c}{ Control } & \multicolumn{2}{c}{ Case } & \multirow{2}{*}{ Total } \\
\cline { 2 - 5 } & Male & Female & Male & Female & \\
\hline$<20$ & 0 & 2 & 0 & 1 & 3 \\
$\mathbf{2 0 - 2 4}$ & 2 & 2 & 1 & 2 & 7 \\
$\mathbf{2 5 - 2 9}$ & 6 & 4 & 3 & 4 & 17 \\
$\mathbf{3 0 - 3 4}$ & 10 & 6 & 9 & 3 & 28 \\
$\mathbf{3 5 - 3 9}$ & 16 & 12 & 9 & 4 & 41 \\
$\mathbf{4 0 - 4 4}$ & 13 & 18 & 12 & 4 & 47 \\
$\mathbf{4 5 - 4 9}$ & 13 & 16 & 11 & 10 & 50 \\
$\mathbf{5 0 - 5 4}$ & 19 & 23 & 21 & 15 & 78 \\
$\mathbf{5 5 - 5 9}$ & 19 & 24 & 12 & 16 & 71 \\
$\mathbf{6 0 - 6 4}$ & 11 & 13 & 18 & 13 & 55 \\
$\mathbf{6 5 - 6 9}$ & 13 & 14 & 18 & 10 & 55 \\
$\mathbf{7 0 - 7 4}$ & 6 & 8 & 12 & 3 & 29 \\
$\mathbf{7 5 - 7 9}$ & 2 & 3 & 4 & 6 & 15 \\
$>\mathbf{7 9}$ & 1 & 0 & 1 & 2 & 4 \\
Total & $\mathbf{1 3 1}$ & $\mathbf{1 4 5}$ & $\mathbf{1 3 1}$ & $\mathbf{9 3}$ & $\mathbf{5 0 0}$ \\
\hline
\end{tabular}

Table 2. Age-matched univariate analysis for epidemiological factors and colorectal cancer

\begin{tabular}{|c|c|c|}
\hline Factor & OR & $95 \% \mathrm{CI}$ \\
\hline Family history of colorectal cancer, 1st degree & 1.48 & $0.69,3.16$ \\
\hline Current Smoker & 1.44 & $0.85,2.44$ \\
\hline Ex-smoker & 1.23 & $0.73,2.07$ \\
\hline Passive smoking & 0.92 & $0.62,1.36$ \\
\hline Current drinker, alcohol & 1.05 & $0.63,1.76$ \\
\hline Ex-drinker, alcohol & 1.06 & $0.62,1.80$ \\
\hline Consumption of canned meat (daily-2/month) & 1.47 & $0.98,2.20$ \\
\hline $\begin{array}{l}\text { Consumption of fish sauce (patis) } \\
\text { (daily-2/month) }\end{array}$ & 1.33 & $0.92,1.94$ \\
\hline $\begin{array}{l}\text { Consumption of shrimp paste (bagoong) } \\
\text { (daily }-2 / \text { month) }\end{array}$ & 1.40 & $0.97,2.02$ \\
\hline Vegetable Consumption ( $\geq 1$ /week) & 0.86 & $0.49,1.49$ \\
\hline $\begin{array}{l}\text { Consumption of scalding hot-food } \\
\text { ( }>5 \text { days } / \text { month) }\end{array}$ & 1.07 & $0.72,1.58$ \\
\hline $\begin{array}{l}\text { Consumption of preserved food (nitrite-treated) } \\
(>5 \text { days/month) }\end{array}$ & 1.34 & $0.87,2.07$ \\
\hline Consumption of smoked food ( $>5$ days/month) & 1.35 & $0.93,1.96$ \\
\hline $\begin{array}{l}\text { Consumption of salted food eater } \\
\text { (>5 days/month) }\end{array}$ & 0.85 & $0.55,1.29$ \\
\hline Moldy food exposure ( $\geq 1 /$ month $)^{a}$ & 1.61 & $1.11,2.35$ \\
\hline Pesticide exposure ( $\geq 1$ /week) & 1.12 & $0.42,2.99$ \\
\hline Vinyl chloride occupational exposure & 2.49 & $0.96,6.47$ \\
\hline Benzene occupational exposure & 2.35 & $1.00,5.50$ \\
\hline UV sunlight exposure ( $\geq 7 /$ month $)^{\mathrm{a}}$ & 1.99 & $1.16,3.39$ \\
\hline Coal carbonization occupational exposure & 2.17 & $0.38,12.38$ \\
\hline Wood dust occupational exposure & 2.66 & $1.21,5.83$ \\
\hline Betel quid chewing & 3.36 & $0.34,33.59$ \\
\hline Oral contraceptive use ( $\geq 1$ / year) & 1.42 & $0.75,2.66$ \\
\hline
\end{tabular}

Source: Ngelangel CA, Javelosa MA, Cutiongco-de la Paz, EM and the Philippine Cancer Genetics Study Group. Epidemiological Risk Factors for Cancers of the Lung, Breast, Colon-rectum \& Oral cavity: A CaseControl Study in the Philippines. Acta Medica Philippina. 2009; 43(4):2934.

${ }^{a}$ Environmental factors that remained significant after age and sex-matched multivariate logistic regression: moldy food exposure OR 2.47 (95\% CI: 1.115.48); UV sunlight exposure OR 1.75 (95\% CI 1.01-3.03); wood dust occupational exposure OR 1.48 (95\% CI: 1.01-2.17)

univariate logistic regression revealed that only the NAT2*6B allele and the NAT2*6B homozygous genotype were found to significantly increase colorectal cancer susceptibility in this study (Table 4).
Table 3. Multivariate logistic regression analyses of genetic and environmental factors

\begin{tabular}{lcc}
\hline \multicolumn{1}{c}{ Variable* $^{*}$} & Odds ratio & OR 95\%CI \\
\hline Constant & -- & -- \\
Age & 1.02 & $1.01,1.04$ \\
UV radiation & 2.08 & $1.21,3.58$ \\
Wood dust exposure & 2.24 & $0.95,5.30$ \\
\hline
\end{tabular}

*The following variables were removed from the model after backward logistic regression: sex, frequency of moldy food exposure and $N A T 2^{*} 6 B$ allele. $N=406 ;-2 L L=542.1 ; R 2=0.05$.

Table 4. Age- and sex-adjusted univariate logistic regression analyses by colorectal cancer status*

\begin{tabular}{lcc}
\hline \multicolumn{1}{c}{ Variable $^{*}$} & Odds ratio (OR) & OR 95\% CI \\
\hline GSTM1 null & 0.95 & $0.66,1.37$ \\
GSTT1 null & 1.08 & $0.75,1.54$ \\
GSTP1 c.313G homozygote & 1.10 & $0.24,4.96$ \\
GSTP1 c.313G allele (dominant) & 1.07 & $0.75,1.53$ \\
GSTP1 c.313G allele (recessive) & 1.06 & $0.59,1.91$ \\
GSTP1 c.313G allele & 1.07 & $0.75,1.53$ \\
$N A T 1 * 3$ allele & 1.58 & $0.61,4.06$ \\
$N A T 1 * 4$ allele & 0.94 & $0.65,1.35$ \\
$N A T 1 * 10$ allele & 1.32 & $0.86,2.05$ \\
$N A T 1 * 11$ allele & 1.09 & $0.39,3.05$ \\
$N A T 2 * 4$ allele & 0.85 & $0.59,1.23$ \\
$N A T 2 * 5 B$ allele & 0.57 & $0.29,1.11$ \\
$N A T 2 * 5 C$ allele & 0.82 & $0.23,2.95$ \\
$N A T 2 * 6 B$ homozygote & $\mathbf{2 . 1 9}$ & $\mathbf{1 . 1 9 , 4 . 0 3}$ \\
$N A T 2 * 6 B$ heterozygote & 1.38 & $0.95,2.02$ \\
$N A T 2 * 6 B$ allele (dominant) & $\mathbf{1 . 5 1}$ & $\mathbf{1 . 0 6 , 2 . 1 6}$ \\
$N A T 2 * 6 B$ allele (recessive) & $\mathbf{1 . 8 7}$ & $\mathbf{1 . 0 5 , 3 . 3 3}$ \\
$N A T 2 * 7 A$ allele & 0.89 & $0.62,1.29$ \\
\hline
\end{tabular}

${ }^{*}$ Formula: Colon cancer status $\sim$ constant $+\beta$ Variable ${ }^{*}$ Variable

\section{Discussion}

Environmental Factors

Among the non-genetic variables, diet is the strongest and most likely contributor to colorectal cancer susceptibility; high intake of meat and fat, and low intake of fruits, vegetables are some dietary factors that have been reported to increase the likelihood of developing this particular cancer type. ${ }^{1,7,14}$ Lifestyle factors such as the lack of physical activity, obesity and alcohol consumption have also been identified as significant risk factors. ${ }^{5,7}$ Recent research has shown that the microbiome in the colon modulates the dietary metabolites that act as carcinogens. ${ }^{8}$ However, we were unable to detect significant interactions between the abovementioned colorectal cancer-associated variables and colorectal cancer risk in our study population; and the environmental factors that were identified to be significant in this study (moldy food exposure, occupational exposure to UV light and wood dust) have not been commonly associated with colorectal cancer, though they have been documented in the past as potential carcinogens and risk modifiers for other cancer types. ${ }^{8,11,33}$ The clinical significance of these findings is uncertain because of the fact that the lower limits of the $95 \%$ CI for the three factors are very close to the null value of one, and also because of the difficulty of 
accurately ascertaining the level of consumption and exposure of subject participants in a retrospective casecontrol study such as this one.

\section{Genetic Factors}

Correlating acetylation status with colorectal cancer risk, the rapid acetylator genotype/phenotype has been hypothesized to cause an increase in colorectal cancer as a result of the higher NAT enzyme activity which activates dietary heterocyclic amines and other carcinogenic intermediates, leading to DNA adduct formation. ${ }^{7,9}$ Studies on animal models have also reported a higher incidence of colon aberrant crypt foci among rapid versus slow acetylators after administration of aromatic amine carcinogens. ${ }^{11}$ Likewise, a number of population-based studies among African Americans, Caucasian, Chinese, Hungarian, Japanese and Portuguese subject participants, as well as one meta-analysis noted a significant excess of NAT2 rapid acetylation genotypes among colorectal cancer patients.4,13,34-37 Two separate meta-analysis studies by Wang et al (meta-analysis of 4 studies of Japanese (2,217 CRC cases, 3,788 controls) and 3 studies of African Americans (527 CRC cases, 4,527 controls) and Ananthakrishnan et al (meta-analysis of 11 studies (8,290 CRC cases and 9,115 controls)), and a study by Procopciuc et al (using 150 Romanian CRC cases and 162 controls; with PCR-RFLP as genotyping method) also showed the association of processed meat and red meat intake to colorectal cancer being significant for patients with NAT2 rapid acetylator phenotypes. ${ }^{4,7,9}$ Sørensen et al found an increase in risk among NAT1 fast acetylators in a prospective study among 379 German CRC cases and 769 sub-cohort members using direct sequencing, while the $N A T 1^{*} 10$ allele-which is suggested to be a rapid acetylator allele that has been noted to increase risk for this cancer type. ${ }^{14,15}$ However, a number of previous studies have been unable to detect any significant interactions between NAT1 and NAT2 genotypes and colorectal cancer susceptibility. ${ }^{6,14,35}$ A systematic review of 73 studies done by Ananthakrishnan et al, also noted that NAT1 polymorphisms have significant association to pancreatic cancer and not colorectal cancer. ${ }^{7}$

The results of this study show that there is no statistically significant relationship between specific NAT1 genotypes and the risk of colorectal cancer in our subject population. Carriers of the $N A T 2 * 6 B$ allele, which results in a slow acetylator phenotype, have demonstrated an increased risk for colorectal cancer. This finding diverges from earlier mentioned reports that linked fast acetylators with increased colorectal cancer risk. After adjustment for non-genetic factors, however, $N A T 2 * 6 B$ allele was dropped from the model. This suggests that the $N A T 2 * 6 B$ allele may not be as significant a modifier of colorectal cancer risk as that of environmental exposure. A recent report, using 150 Romanian CRC cases with PCR-RFLP and sequencing as genotyping methods, has shown that $N A T 2 * 6 B$ allele increases risk for sporadic colorectal cancer $(\mathrm{OR}=5.16$; $\mathrm{p}<$ 0.0001) among Romanians. ${ }^{9}$

As for the GSTs, the null GSTM1 and GSTT1 genotypes, and the GSTP1 c.313A>G polymorphism was evaluated as risk modifiers for colorectal cancer. The c.313A $>\mathrm{G}$ transition in GSTP1 results in an isoleucine to valine substitution at codon 105, close to the enzyme's binding site for electrophilic substrates. ${ }^{26}$ The $105 \mathrm{val}$ allele has also been associated with reduced GSTP1 enzyme activity. ${ }^{27}$ Similarly, reduced detoxification activity is also expected from the homozygous deletion of GSTM1 or GSTT1, since the null genotype for either gene leads to a lack of catalytic activity of their respective enzyme products. ${ }^{19,24}$ All three GST variants have been hypothesized to increase colorectal cancer susceptibility as a result of the slower deactivation rate of potential carcinogens.

Studies have shown that the GSTM1 null variant increases cancer risk among Caucasian, Chinese, Indian, Kazakh, Romanians, Russian, Saudi Arabians, Spanish and Turkish populations; while the GSTT1 homozygous deletion has also been identified as a significant genetic risk factor for Caucasian, Chinese, Indian, Spanish and Turkish population-based studies. ${ }^{9,10,20-22,38-43}$ In two separate metaanalyses done by Li et al (using 33 studies with 8,502 CRC cases and 13,699 controls) and Liu et al (using 19 articles containing 3,217 cases), the null genotype of GSTM1 was significantly associated with susceptibility to colorectal cancer and neurotoxicity, respectively, in Asians. ${ }^{23,24}$ It was also shown to be associated with poor overall survival and poor disease-free survival. ${ }^{25}$ The heterozygous genotype of GSTT1 was seen to be correlated with cancer susceptibility in Kazakh and Russian populations $(\mathrm{OR}=1.98 ; 95 \% \mathrm{CI}=1.30$ 3.00; $\mathrm{p}=0.001) \cdot{ }^{21,25}$ Additionally, the combination of the null genotypes of GSTM1 and GSTT1 increases the risk of colorectal cancer among Chinese (OR, 1.95; 95\% CI, 1.33-2.85; $\mathrm{p}<0.001$ ), ethnic Kashmiri (OR = 3.5, 95\% CI: 0.9-13.8; $\mathrm{p}=$ $0.06)$ and Pakistani populations. ${ }^{10,17,19}$ Then again, the systematic review done by Liu et al also concluded that GSTM1 and GSTT1 have no association with chemotherapyrelated toxicities and tumor response. ${ }^{24}$

Among the three GST genes, GSTP1 in particular, is a potential biomarker because it is widely expressed in the colon. ${ }^{39}$ However, reports on the effect of the GSTP1 c.313G on colorectal cancer risk have been inconsistent; with one study identifying the homozygous variant genotype as a risk factor, while another study finding the homozygous variant genotype to be protective. ${ }^{32,37}$ However, many other molecular epidemiology reports have also found no statistically significant associations with polymorphisms in the GSTM1, GSTP1, or GSTT1 genes with colorectal cancerthese is similar to the findings of our own study among Filipino subject participants. ${ }^{10,18,20,21,24,34,41,43,44}$ The association of GSTP1 c.313G to tumor response, progression and overall 
survival after chemotherapy in colorectal and gastric cancers have also been demonstrated in a systematic review by Shen et al in 2016 but this was not part of this study. ${ }^{27}$

As a case-control epidemiological study, there are inherent methodological limitations particularly pertaining to the evaluation of non-genetic factors as markers of colorectal cancer risk in the study population. Although the study was able to detect novel interactions between environmental variables and cancer susceptibility, a comprehensive prospective study coupled with a refinement in data collection techniques are needed to validate the findings.

For the genetic factors, the lack of significant associations does not necessarily discount the potential of these metabolic gene polymorphisms as colorectal cancer biomarkers. A more comprehensive study having a greater population size and allelic/genotype coverage of the candidate genes is recommended to better understand the individual and combinatorial effects of the different gene variants on cancer risk. To evaluate the interaction of environmental and genetic factors, stratified analysis with regard to epidemiologic subgroups is suggested in order to better identify at-risk subgroups in the population.

\section{Conclusion}

Among the non-genetic factors investigated in the study, only exposure to moldy food, occupational exposure to wood dust as well as to UV sunlight were found to increase susceptibility to colorectal cancer. As regards genetic polymorphisms in the xenobiotic metabolizing enzyme genes, only the $N A T 2 * 6 B$ allele and $N A T 2 * 6 B / N A T 2 * 6 B$ genotype were found to be a significant modifier of disease risk. Multivariate analysis of both environmental and genetic factors, however, suggests that UV sunlight and wood dust exposure play a greater role in influencing colorectal cancer susceptibility than NAT2 genotype status. A comprehensive prospective study using a greater population size and allelic/genotype coverage of the candidate genes is recommended to validate the findings and to provide more insights on the gene-environment and gene-gene interactions of the environmental variables and different gene variants on colorectal cancer risk.

\section{Acknowledgments}

We acknowledge the individuals who worked on this project: Josef Misael Cortez, Feorillo Galivo, Ma. Rosanna Mendoza, Cindy Oarmata, Rhocile Lee Romblon, Gloriamaris Loy, Madonna Balbacal, Anna Kristina Serquina, Jane Mercado, Joren Bumacod, Edgar Tafaleng, Marife Arancillo, Fang-I Chao, Noelle Niña Gianan, William Augustine Roque, Iris Diana Uy, Geralyn Valleza, Julio Rodrigo Reyes, Jo Erika Narciso, Diana Victoria Doromal, Noel Francis Javellana, Nicie Gayle Fabros, Karl Cabalteja, and Francis Tablizo.

\section{Statement of Authorship}

All authors have approved the final version submitted.

\section{Author Disclosure \\ All authors declared no conflict of interest.}

\section{Funding Source}

The grant support for this research was provided by the Philippine Council for Health Research and Development, Department of Science and Technology and the Institute of Human Genetics, National Institutes of Health, University of the Philippines Manila.

\section{References}

1. Boyle P. International Agency for Research on Cancer. World cancer report 2008. Lyon: IARC Press; 2008.

2. Ferlay J, Shin HR, Bray F, Forman D, Mathers C, Parkin DM. GLOBOCAN 2008, Cancer Incidence and Mortality Worldwide: IARC CancerBase No. 10 Lyon, France: International Agency for Research on Cancer [Online]. 2010 [cited 2010 Jan]. Available from http://globocan.iarc.fr.

3. Afinidad-Bernardo D. Colorectal cancer now Philippines' number 1 cancer [Online]. [cited 2017 Mar]. Available from http:/ www.philstar.com/health-and-family/2017/03/08/1679163/colorectalcancer-now-philippines-number-1-cancer.

4. Wang $\mathrm{H}$, Iwasaki M, Haiman $\mathrm{CA}$, et al. Interaction between red meat intake and NAT2 Genotype in increasing the risk of colorectal cancer in Japanese and African Americans. PLoS One. 2015; 10(12):e0144955. doi: 10.1371/journal.pone.0144955.

5. Andersen V, Vogel U. Interactions between meat intake and genetic variation in relation to colorectal cancer. Genes Nutr. 2015; 10(1):448. doi: 10.1007/s12263-014-0448-9.

6. Zhang K, Gao L, Wu Y, et al. NAT1 polymorphisms and cancer risk: a systematic review and meta-analysis. Int J Clin Exp Med. 2015; 8(6):9177-91.

7. Ananthakrishnan AN, Du M, Berndt SI, et al. Red meat intake, NAT2, and risk of colorectal cancer: a pooled analysis of 11 studies. Cancer Epidemiol Biomarkers Prev. 2015; 24(1):198-205. doi: 10.1158/10559965.EPI-14-0897.

8. Rattray NJW, Charkoftaki G, Rattray Z, Hansen JE, Vasiliou V, Johnson $\mathrm{CH}$. Environmental influences in the etiology of colorectal cancer: the premise of metabolomics. Curr Pharmacol Rep. 2017; 3(3):114-25. doi: 10.1007/s40495-017-0088-z.

9. Procopciuc LM, Osian G, Iancu M. Colorectal cancer carcinogenesis: a multivariate genetic model in a cohort of Romanian population. Clin Lab. 2017; 63(4):647-58. doi: 10.7754/Clin.Lab.2016.160821.

10. Cong N, Liu L, Xie Y, Shao W, Song J. Association between glutathione Stransferase T1, M1, and P1 genotypes and the risk of colorectal cancer. J Korean Med Sci. 2014; 29(11):1488-92. doi: 10.3346/jkms.2014. 29.11.1488.

11. Hein DW. Molecular genetics and function of NAT1 and NAT2: role in aromatic amine metabolism and carcinogenesis. Mutat Res. 2002; 506507:65-77.

12. Minchin RF, Hanna PE, Dupret JM, Wagner CR, Rodrigues-Lima F, Butcher NJ. Arylamine N-acetyltransferase I. Int J Biochem Cell Biol. 2007; 39(11):1999-2005.

13. Butler LM, Millikan RC, Sinha R, et al. Modification by $\mathrm{N}$ acetyltransferase 1 genotype on the association between dietary heterocyclic amines and colon cancer in a multiethnic study. Mutat Res. 2008; 638(1-2):162-74.

14. Sørensen M, Autrup H, Olsen A, Tjønneland A, Overvad K, RaaschouNielsen O. Prospective study of NAT1 and NAT2 polymorphisms, tobacco smoking and meat consumption and risk of colorectal cancer. 
Cancer Lett. 2008; 266(2):186-93.

15. Montazeri Z, Theodoratou E, Nyiraneza C, et al. Systematic metaanalyses and field synopsis of genetic association studies in colorectal adenomas. Int J Epidemiol. 2016; 45(1):186-205. doi: 10.1093/ije/dyv185

16. Liu F, Ji F, Ji Y, et al. In-depth analysis of the critical genes and pathways in colorectal cancer. Int J Mol Med. 2015; 36(4):923-30. doi: 10.3892/ijmm.2015.2298.

17. Nissar S, Sameer AS, Rasool R, Chowdri NA, Rashid F. Evaluation of deletion polymorphisms of glutathione S-transferase genes and colorectal cancer risk in ethnic Kashmiri population: A case-control study. Indian J Cancer. 2016; 53(4):524-8. doi: 10.4103/ijc.IJC_17_17.

18. Gorukmez O, Yakut T, Gorukmez O, et al. Glutathione S-transferase T1, M1 and P1 genetic polymorphisms and susceptibility to colorectal cancer in Turkey. Asian Pac J Cancer Prev. 2016; 17(8):3855-9.

19. Masood N, Mubashar A, Yasmin A. Epidemiological factors related to GSTM1 and GSTT1 genes deletion in colon and rectum cancers: A casecontrol study. Cancer Biomark. 2015; 15(5):583-9. doi: 10.3233/CBM150498 .

20. Djansugurova L, Zhunussova G, Khussainova E, et al. Association of DCC, MLH1, GSTT1, GSTM1, and TP53 gene polymorphisms with colorectal cancer in Kazakhstan. Tumour Biol. 2015; 36(1):279-89. doi: 10.1007/s13277-014-2641-2. Epub 2014 Sep 24.

21. Zhunussova G, Zhunusbekova B, Djansugurova L. Association between glutathione S-transferase M1 and T1 polymorphisms and colorectal cancer risk in patients from Kazakhstan. Clin Lab. 2015; 61(1-2):161-8.

22. Khabaz MN, Nedjadi T, Gari MA, et al. GSTM1 gene polymorphism and the risk of colorectal cancer in a Saudi Arabian population. Genet Mol Res. 2016; 15(1). doi: 10.4238/gmr.15017551.

23. Li J, Xu W, Liu F, Huang S, He M. GSTM1 polymorphism contribute to colorectal cancer in Asian populations: a prospective meta-analysis. Sci Rep. 2015; 5:12514. doi: 10.1038/srep12514.

24. Liu H, Shi W, Zhao L, Dai D, Gao J, Kong X. Can GSTM1 and GSTT1 polymorphisms predict clinical outcomes of chemotherapy in gastric and colorectal cancers? A result based on the previous reports. Onco Targets Ther. 2016; 9:3683-94. doi: 10.2147/OTT.S105158.

25. Yan S, Wang Z, Wang Z, et al. Glutathione S-transferase M1 null genotype related to poor prognosis of colorectal cancer. Tumor Biol. 2016; 37(8):10229-34. doi: 10.1007/s13277-015-4676-4.

26. Khabaz MN, Al-Maghrabi JA, Nedjadi T, et al. Does Val/Val genotype of GSTP1 enzyme affects susceptibility to colorectal cancer in Saudi Arabia? Neuro Endocrinol Lett. 2016; 37(1):46-52.

27. Shen X, Wang J, Yan X, et al. Predictive value of GSTP1 Ile105Val polymorphism in clinical outcomes of chemotherapy in gastric and colorectal cancers: a systematic review and meta-analysis. Cancer Chemother Pharmacol. 2016 77(6):1285-302. doi: 10.1007/s00280-0163047-1.

28. Sreelekha TT, Ramadas K, Pandey M, Thomas G, Nalinakumari KR, Pillai MR. Genetic polymorphism of CYP1A1, GSTM1 and GSTT1 genes in Indian oral cancer. Oral Oncol. 2001; 37(7):593-8.

29. To-Figueras J, Gené M, Gómez-Catalán J, et al. Glutathione S-transferase M1 (GSTM1) and T1 (GSTT1) polymorphisms and lung cancer risk among Northwestern Mediterraneans. Carcinogenesis. 1997; 18(8):1529-33.
30. Watson MA, Stewart RK, Smith GB, Massey TE, Bell DA. Human glutathione S-transferase P1 polymorphisms: relationship to lung tissue enzyme activity and population frequency distribution. Carcinogenesis. 1998;19(2):275-80.

31. Bell DA, Stephens EA, Castranio $\mathrm{T}$, et al. Polyadenylation polymorphism in the acetyltransferase 1 gene (NAT1) increases risk of colorectal cancer. Cancer Res. 1995; 55(16):3537-42.

32. Hubbard AL, Harrison DJ, Moyes C, et al. N-acetyltransferase 2 genotype in colorectal cancer and selective gene retention in cancers with chromosome 8p deletions. Gut. 1997; 41(2):229-34.

33. Rekhadevi PV, Mahboob M, Rahman MF, Grover P. Genetic damage in wood dust-exposed workers. Mutagenesis. 2009; 24(1):59-65.

34. Cotterchio M, Boucher BA, Manno M, Gallinger S, Okey AB, Harper PA Red meat intake, doneness, polymorphisms in genes that encode carcinogen-metabolizing enzymes, and colorectal cancer risk. Cancer Epidemiol Biomarkers Prev. 2008;17(11):3098-107.

35. Kiss I, Németh A, Bogner B, et al. Polymorphisms of glutathione-S transferase and arylamine $\mathrm{N}$-acetyltransferase enzymes and susceptibility to colorectal cancer. Anticancer Res. 2004; 24(6):3965-70.

36. Gil JP, Lechner MC. Increased frequency of wild-type arylamine-Nacetyltransferase allele NAT2* 4 homozygotes in Portuguese patients with colorectal cancer. Carcinogenesis. 1998; 19(1):37-41.

37. Chen $\mathrm{K}$, Jin $\mathrm{MJ}$, Fan $\mathrm{CH}$, et al. [A case-control study on the association between genetic polymorphisms of metabolic enzymes and the risk of colorectal cancer]. Zhonghua Liu Xing Bing Xue Za Zhi. 2005; 26(9):659-64.

38. Martinez C, Martin F, Fernandez JM, et al. Glutathione S-transferases 1, theta1, pi 1, alpha 1 and mu 3 genetic polymorphisms and the risk of colorectal and gastric cancers in humans. Pharmacogenomics. 2006;7(5):711-8.

39. Ateş NA, Tamer L, Ateş C, et al. Glutathione S-transferase M1, T1, P1 genotypes and risk for development of colorectal cancer. Biochem Genet. 200543(3-4):149-63.

40. Economopoulos KP, Sergentanis TN. GSTM1, GSTT1, GSTP1, GSTA1 and colorectal cancer risk: a comprehensive meta-analysis. Eur J Cancer. 201046(9):1617-31.

41. Wang J, Jiang J, Zhao Y, et al. Genetic polymorphisms of glutathione Stransferase genes and susceptibility to colorectal cancer: a case-control study in an Indian population. Cancer Epidemiol. 2011; 35(1):66-72.

42. Butler WJ, Ryan P, Roberts-Thomson IC. Metabolic genotypes and risk for colorectal cancer. J. Gastroenterol. Hepatol. 2001; 16(6):631-5.

43. Yeh CC, Sung FC, Tang R, Chang-Chieh CR, Hsieh LL. Association between polymorphisms of biotransformation and DNA-repair genes and risk of colorectal cancer in Taiwan. J Biomed Sci. 2007; 14(2):183-193.

44. Shahnam A, Ridha Z, Wiese MD, Kichenadasse G, Sorich MJ. Pharmacogenetic and ethnicity influence on oxaliplatin therapy for colorectal cancer: a meta-analysis. Pharmacogenomics. 2016; 17(15):1725-32. 